\title{
Utilization of Abdominal Ultrasonography in AIDS Patients
}

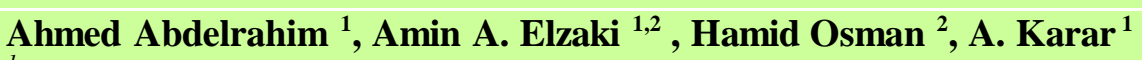 \\ ${ }^{l}$ Alzaiem Alazhari University, Faculty of Radiological Sciences \& Medical Imaging, \\ Sudan \\ ${ }^{2}$ Taif University, College of Applied Medical Science, Radiological Science \\ Department, KSA
}

Corresponding Author Amin A. E. Elzaki

Mobile:

$+966533821879$

\section{E mail:}

Aminzaki2000@yahoo. com

Key words: AIDS, Abdominal Ultrasonography, HIV
Background and study aim: The acquired immunodeficiency syndrome AIDS is a destructive disease, which become a major out-break in the community affecting both adult and young people, causing not only ill-health but affect both the economy, and the psychology of the patient. The abdomen is the largest cavity in the body. Ultrasound is most cheap, available and non invasive tool that can be used in diagnosing abdominal diseases. The aim of the current study was to detect the ultrasonographic findings of prevalent non-specific abdominal abnormalities associated with AIDS.

Patients and Methods: 200 patients with confirmed positive HIV were scanned at the Voluntary and Counseling Testing Centre in Omdurman Teaching Hospital, Sudan. with an international scanning guideline and protocols for abdominal ultrasonography. A two ultrasound

\section{INTRODUCTION}

The acquired immunodeficiency syndrome AIDS is a retroviral disease caused by the human immunodeficiency virus HIV and is characterized by the profound immuno suppression leading to opportunistic infections, secondary neoplasms, and neurologic manifestation [1,2].

In the past decade, AIDS has become a major health problem throughout the world. The world Health Organization WHO statistics showed that on $1^{\text {st }}$ of July 1989 there were 167373 patients reported to have full blown AIDS. Of these, 95561 were in the United State of America USA, 29906 in Africa and 22708 in Europe. It estimated that scanners (Shimadzu SDU-350XL and KIAXIN) were used for scanning.

Results: The most affected age group was the $4^{\text {th }}$ decade $(36 \%)$ which were in the active and product period and most of them $(72 \%)$ were married. The most frequent abdominal ultrasonography findings were splenomegaly, enlarged lymph nodes, hepatomegaly and increased liver echogenicity $(31.25 \%)$, (13.54\%), $(43.75 \%),(26.04 \%)$ respectively. The low frequent occurring were focal lesions in parenchymal of the organs and nephromegaly $(1.04 \%)$, ascites $(4.17 \%)$, portal hypertension $(1.04 \%)$ and abscesses (1.04\%).

Conclusion: Abdominal ultrasonography revealed that $(48.0 \%)$ of the patients developed abnormal abdominal findings supporting the use of abdominal ultrasonography as a diagnostic tool in AIDS.

there are 5 million people in the world who are infected [3].

In 2009, 33.3 million people were living with HIV worldwide, 2.6 million people newly infected with HIV worldwide, and 1.8 million people died of AIDS -related illness worldwide[4,5].

In the united states, the typical adult patient with AIDS present with fever, weight loss, diarrhea, generalized lymphadenopathy, multiple opportunistic infections, neurologic disease, and (in many cases) secondary neoplasms [1]. 
Although the largest number of infections are in Africa, the most rapid increase in HIV infection in past decade are in south east Asian countries, including Thailand, India and Indonesia[1].

The annual number of new HIV infections has been steadily declining since the late 1990s and there are fewer AIDS-related deaths due to the significant scale up of antiretroviral therapy over the past few years. Although, the number of new infections has been falling, level of new infections overall is still high, and with significant reductions in mortality the number of people living with HIV worldwide has increase[6].

In Sudan (September 2008) estimated number of adults and children living with HIV whether or not they have developed symptoms of AIDS as follows: adults $>15$ years represented 270.000 VS children < 14 years represented 20.000 [4].

Patients with AIDS have a high incidence of certain tumors, particularly Kaposi sarcoma, non-Hodgkin lymphomas, and cervical cancer in women [1].

From the epidemiologic and subsequent laboratory investigations the transmission of HIV occurs under conditions that facilitate the exchange of blood or body fluids that contain the virus or virus-infected cells. Thus, the three major routes are sexual contact, parenteral inoculation, and passage of virus from infected mothers to their newborn [1].

Diagnostic ultrasonography imaging provides; a dynamic means of evaluating abdominal soft tissue structures in cross section [7] and also provides information concerning the size, shape, and echo pattern, position of the organs and other structure[8].

Areas affected by AIDS include central nervous system (limitation of ultrasound imposed by bones), respiratory system (ultrasound imposed by air in lung), abdominal retroperitoneal and superficial. Sonographic findings of prevalent non-specific abdominal abnormalities associated with AIDS include splenomegaly, hepatomegaly hyperechoic liver parenchyma, gall bladder wall thickening, lymphadenopathy, and nephropathy [9].

Nephropathy associated with HIV infection is an important cause of AIDS morbidity. Sonographically the characteristic findings are enlarged kidneys with increased cortical echogenicity. Additional findings include a globular appearance of the kidneys, decreased renal sinus fat, and heterogeneous parenchyma with echogenic striations [10].

Acute pancreatitis is common in AIDS caused by various opportunistic infections and medication; develop in $25 \%$ of patients taking didanosve. If patient is known to have AIDS, careful scanning will sometimes reveal echogenic thickening of the distal duct wall indicative of a stricture due to AIDS cholangitis [11].

The most common splenic ultrasonographic finding in AIDS is moderate splenomegaly, reported with $50 \%$ to $70 \%$ of patients referred for abdominal ultrasonography. Focal lesions can occur in AIDS. These may be caused by opportunistic infections such as candida, neumocystis, or mycobacterium. There have been reports of disseminated pneumocystis appearing as tiny focal echoes through the liver, spleen, and kidneys. The spleen may also be involved in Kaposi's sarcoma or lymphoma. Although fluid filled, actively peristaltic gut may be seen with infectious viral or bacterial gastroenteritis. Most affected patients do not demonstrate a sonographic abnormality. Also, certain high-risk populations, such as those with AIDS and neutropenia, appear to be susceptible to acute typhlitis and colitis, which also have a highly suggestive sonographic appearance [11].

\section{OBJECTIVES}

The aim of the study is to detect the findings of abdominal abnormalities associated with AIDS.

\section{MATERIALS AND METHODS}

This is a descriptive study concerns with the abdominal ultrasonographic findings in AIDS. The study was conducted at the Voluntary and Counseling Testing Centre in Omdurman Teaching Hospital, Sudan. The center is supplied by WHO and concern with HIV/AIDS offering psychological treatment, antiretroviral therapy, laboratory investigations and follow up.

A simple random sampling technique selected a number of two hundred confirmed positive HIV with signs and symptoms of AIDS were scanned by ultrasound. Those patients who have not developed AIDS signs and symptoms were excluded from participation. All participants were infectious and treated with different anti viral drugs and medications as well. All patients 
were scanned twice according to the international protocol and guidelines of abdominal ultrasonography scanning [9]. The first scan was performed by the researchers and the findings were confirmed by a consultant radiologist.

The abdomen was completely evaluated in at least two scanning planes. Surveys were used to set correct imaging techniques, to rule out pathologies, and to recognize any normal variants. Typically, full abdominal surveys begin with aorta, followed by the inferior vena cava and the liver, and then the rest of the abdominal organs and associated structures. Only if they are well visualized, survey the aorta along the left lobe of the liver and the inferior vena cava with right lobe followed by the rest of the abdominal organs and associated structures. If an abnormality is identified, it is surveyed in at least two scanning planes following the completed survey of the abdominal organs.

The following criteria were utilized to assess the abdominal organs: Hepatomegaly- longitudinal dimension at mid-claviular line $>15 \mathrm{~cm}$; splenomegaly- longitudinal dimension $>13 \mathrm{~cm}$; thickened gallbladder wall- dimension $>3 \mathrm{~mm}$ at the anterior wall ; pancreatic enlargementdimension $>1.5 \mathrm{~cm}, 2,5 \mathrm{~cm}$ or $2.0 \mathrm{~cm}$ for the head, body or tail respectively; renomegalylongitudinal dimension $>12 \mathrm{~cm}$. thickened bowel wall- thickness $>4 \mathrm{~mm}$; biliary dilatationintrahepatic biliary ducts luminal diameter > $2 \mathrm{~mm}$, common bile duct > $6 \mathrm{~mm}$; lymphadenopathy- visualized lymph nodes (paraaortic) measured $>10 \mathrm{~mm}$. The ultrasonographic criteria of enlargement of mesenteric lymphnodes has been variably defined as the detection of nodes larger than $4 \mathrm{~mm}$ in the short axis and larger than $10 \mathrm{~mm}$ in the long axis.

For scanning a Shimadzu SDU- 350XL (Japan) ultrasound machine with multi-frequency curvilinear probe $(3.5-5 \mathrm{MHz})$ which has variable focal zone and frequency capability, and
KIAXIN (China) with two probes curvilinear multi-frequency $(2 \mathrm{MHz}-5 \mathrm{MHz})$ and linear high frequency $6.5 \mathrm{MHz}$ probe have been used. High frequency probe $6.5 \mathrm{MHz}$ was used to evaluate the gallbladder, the abdominal wall, appendix and other superficial structures. Shimadzu SDU-350 Xl curvilinear probe was used for the other abdominal organs. Proper setting of the overall gain system and time gain or depth gain compensation (TGC/ DGC) was adjusted to optimally visualize each organ.

To ensure combined validity and reliability the ultrasound results was verified by consultant radiologist who had expertise in performing ultrasound scanning. To ensure generalizability all patients were scanned by the same ultrasound machine using the same international guidelines and protocol for performing ultrasound and with using the same room preparation, then it is assumed that all patients offered the same level performing ultrasound.

Especial consideration was given to the right confidentially and anonymity of all research participants. Anonymity was achieved by using numbers for each research participant that would provide link between the information collected and the participants. In addition confidentiality was ensured by making the collected data accessible only to the researcher and the consultant radiologist. The right to equality will be ensured by giving each patient the same facilities, and the privacy of each patient was considered, so no individual patient's details throughout this study. Justice and human dignity was observed by treating selected patients equally when telling them to participate in the research as a sample of this study. The patients were free to decide whether to participate or not. Permission to conduct study was obtained from the hospital director of Omdurman Teaching Hospital. Data has been analyzed using SPSS ( V.16) and presented as number and percentages. 


\section{RESULTS}

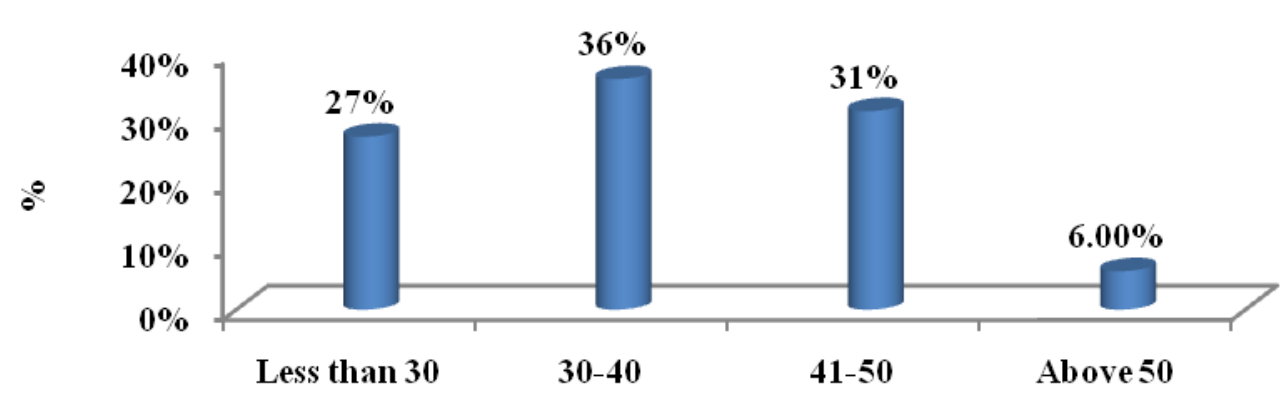

Age group

Figure (1): Distribution of patients according to age

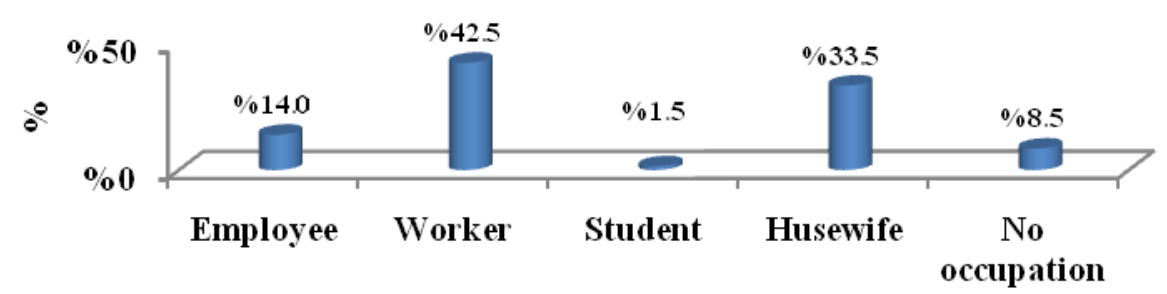

Figure (2): Distribution of patients according to occupation

Table (1): Frequency distribution of ultrasonography concerning liver size

\begin{tabular}{|l|c|c|}
\hline \multicolumn{1}{|c|}{ Liver size } & No. & Percentage \% \\
\hline Normal & 54 & 56.25 \\
\hline Enlarged (Hepatomegaly) & 42 & 43.75 \\
\hline Total & 96 & 100 \\
\hline
\end{tabular}


Table (2): Frequency distribution of ultrasound findings concerning liver in AIDS patients

\begin{tabular}{|l|c|c|}
\hline \multicolumn{1}{|c|}{ Findings } & No. & Percentage \% \\
\hline Paraenchyma echotexture & 1 & 1.04 \\
\hline Hemangioma & 1 & 1.04 \\
\hline Pyogenic abscess & 1 & 1.04 \\
\hline Calcification & 1 & 1.04 \\
\hline Simple cyst & 2 & 2.04 \\
\hline $\begin{array}{l}\text { Coarse liver parenchyma echotexture, hemangioma, liver } \\
\text { abscess and calcification }\end{array}$ & 90 & 93.76 \\
\hline Total & 96 & 100 \\
\hline
\end{tabular}

Table (3): Frequency distribution of ultrasound findings concerning size of spleen

\begin{tabular}{|l|c|c|}
\hline \multicolumn{1}{|c|}{ Spleen size } & No. & Percentage \% \\
\hline Normal & 66 & 68.75 \\
\hline Splenomegaly & 30 & 31.25 \\
\hline Total & 96 & 100 \\
\hline
\end{tabular}

Table (4): Frequency distribution of ultrasound findings concerning spleen echogenicity

\begin{tabular}{|l|c|c|}
\hline \multicolumn{1}{|c|}{ Spleen echogenicity } & No. & Parentage \% \\
\hline Normal & 88 & 91.67 \\
\hline Decreased & 3 & 3.12 \\
\hline Increased & 5 & 5.21 \\
\hline Total & 96 & 100 \\
\hline
\end{tabular}

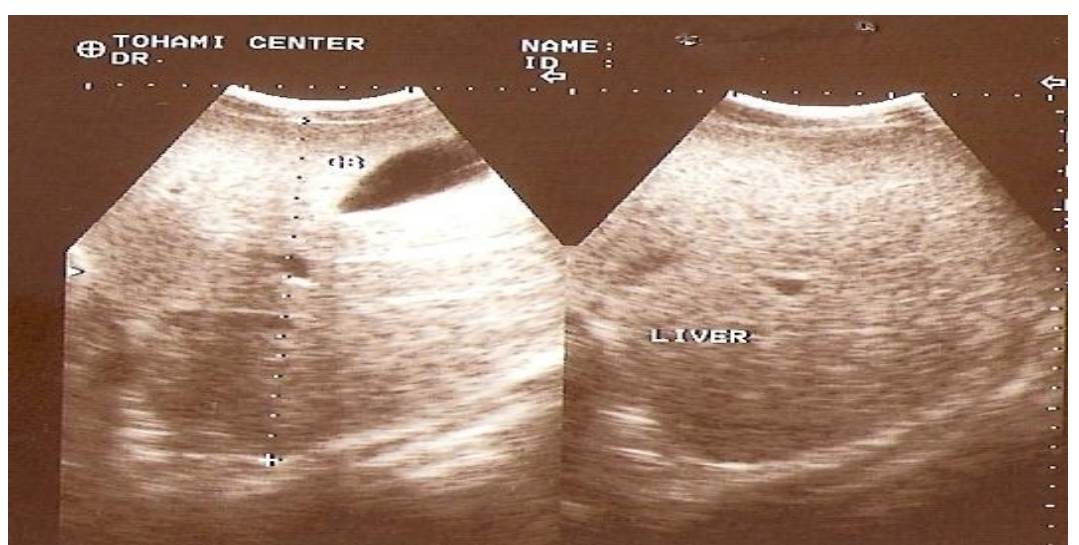

Image (1): A longitudinal gray scale sonogram of the liver of 42 years old male AIDS patient showing hepatomegaly.

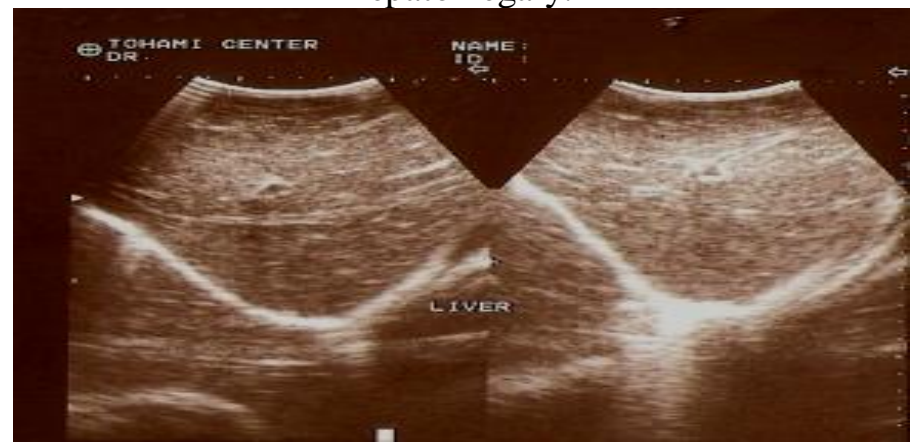

Image (2): A longitudinal sonogram of the liver of 40 years old male AIDS patient showing coarse liver parenchyma. 


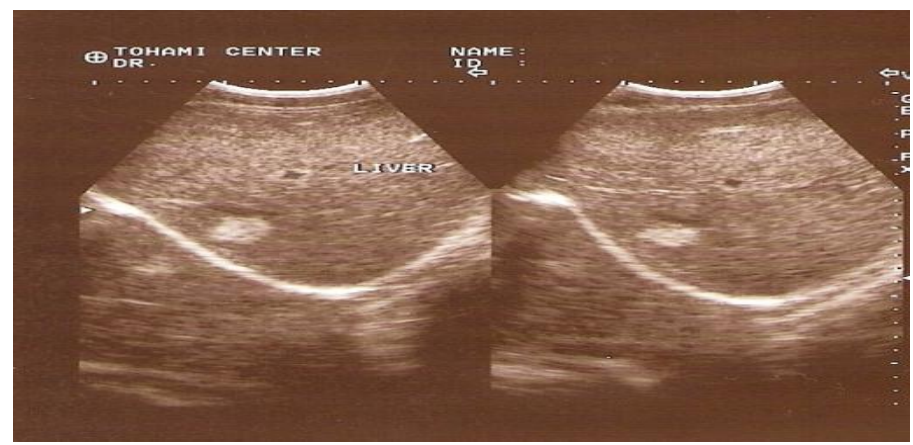

Image (3): A longitudinal sonogram of the liver of 33 years old male AIDS patient showing solid hyperechoic mass (hemangioma).

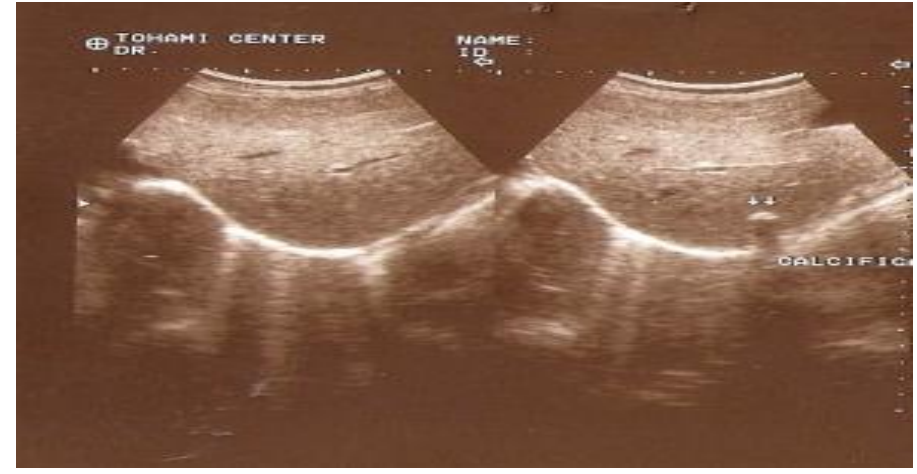

Image (4): A longitudinal gray scale sonogram of the liver showed small calcification.

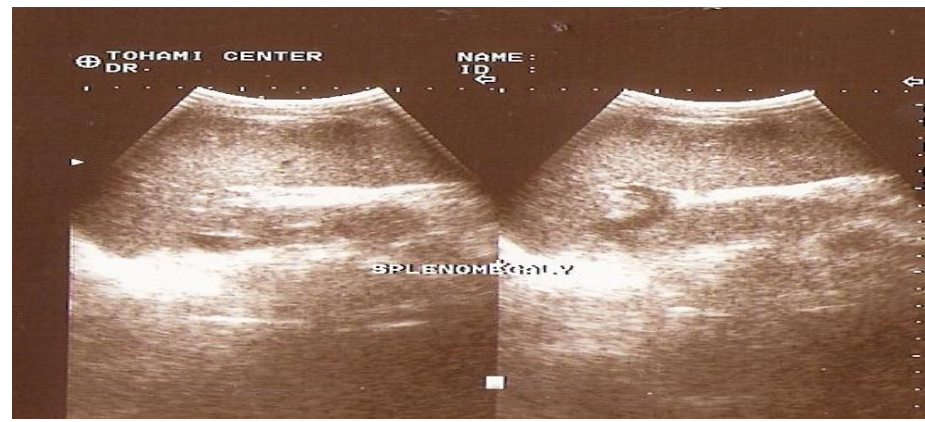

Image (5): A longitudinal sonogram of a 22 years old male AIDS patient showing splenomegaly

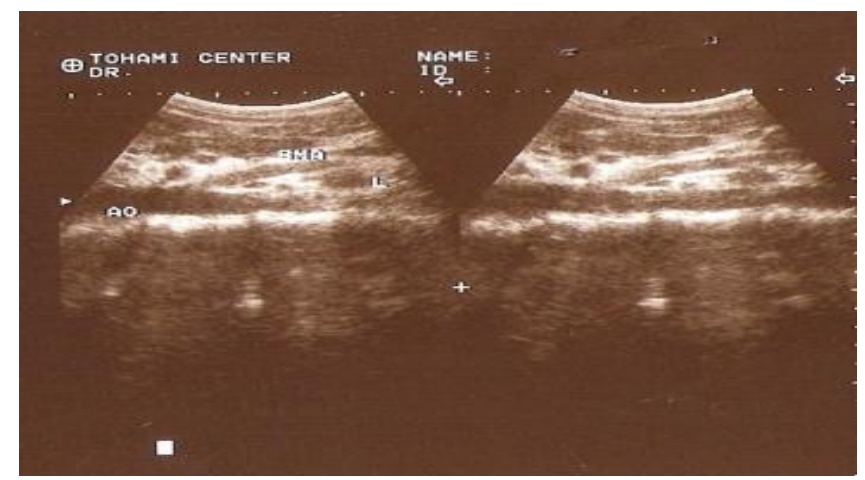

Image (6): A sagittal sonogram of 35 years male AIDS patient showing enlarged paraortic lymph nodes. 


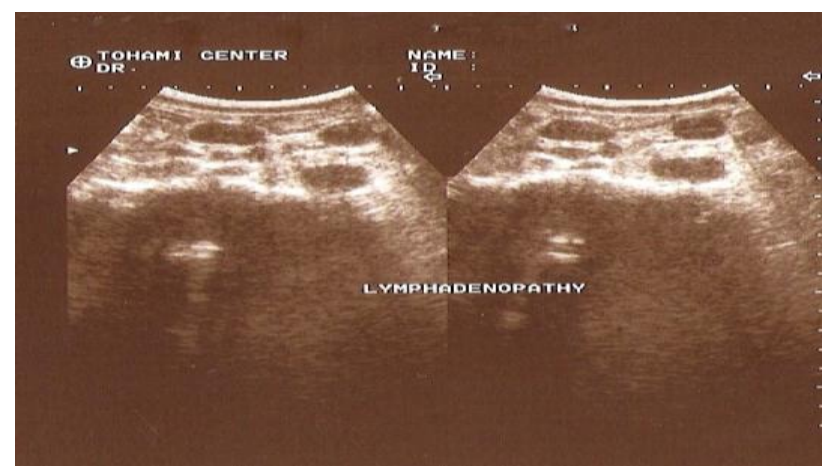

Image (7): A gray scale sonogram of 25 years old female AIDS patient showing enlarged lymph nodes.

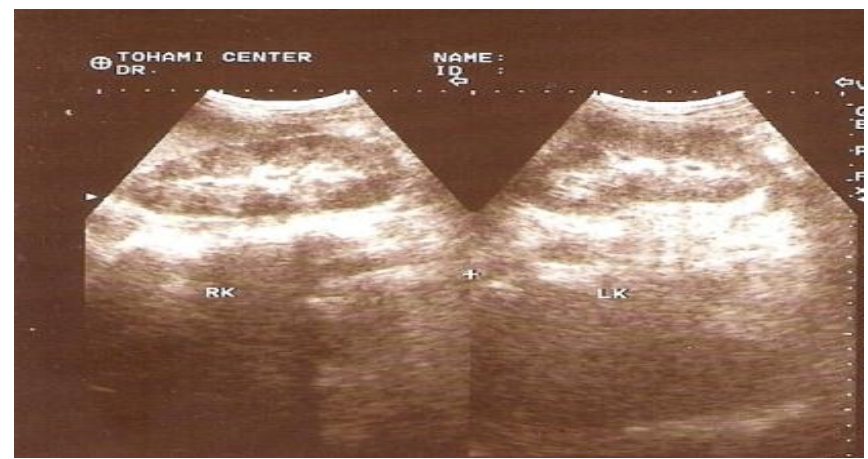

Image (8): A longitudinal sonogram of a 25 years old male AIDS patient showing bilateral increased renal cortex echogenicity.

\section{DISCUSSION}

Two hundred patients were scanned, 109 patients were male forming $(54.50 \%)$. This showed slight difference from Obajami et al who studied abdominal ultrasonography in HIV/AIDS patients in southern Nigeria [2]. Obajami et al [2] revealed that $(66.5 \%)$ were females and $(33.5 \%)$ were males, this difference may be due to the tradition state of our community and how people were dealing with AIDS patient.

The age parameter was divided into four groups in this study < 30years, between 31 up to 40 years, between 41 up to 50 years, and $>50$ years. AIDS was most prevalent in the $4^{\text {th }}$ decade or the most effected age group was between $31-40$ years, this forms about (36\%). The finding matches with Obajimi et al [2] who reported that the prevalence of the disease was in the $4^{\text {th }}$ decade confirming the theory that prove the most common cause of HIV transmission increases due to increasing sexual activity in the $3^{\text {rd }}$ and $4^{\text {th }}$ decade [2].

The majority of the patients were workers forming $(42.5 \%)$ of the participants. This clearly explain that low level of education increases the possibility of an individual to be infected by HIV.

In this study positive ultrasonography findings represent (48\%) of the two hundred participants suggests that this record support the use of ultrasonography in AIDS management program and make it a reliable modality as an imaging tool.

Hepatomegaly was the most liver finding by ultrasonography. It was demonstrated in (43.75\%) of the patients showing mostly non specific findings such as increased liver parenchyma echogenicity (26.04\%) compatible 
with fatty infiltration of the liver. Tshibwabwa et al [12] recorded that patients had hepatomegaly were (35\%). Slightly lowering percentage by Tshibwabwa et al may be due to increased risk factors of hepatomegaly in our area because of malaria.

Other liver findings include coarse liver parenchyma echotexture, hemangioma, liver abscess, and calcification representing the same incidence (1.04\%). Furthermore, (2.04\%) patients had simple cysts. The only solid hyperechoic mass recorded was not biopsied for lack of appropriate facility in the centre.

Hepatosplenomegaly in AIDS patients in the absence of hepatic focal lesions may suggest infection (M. avium intracellular, malaria or histoplasmosis) rather than lymphoma [13].

Yee et al attributed the diffuse infiltration or increased hepatic echogenicity mostly to fatty infiltration or hepatic granulomatosis [14]. However, hepatitis from infection or drugs can cause the observed hyperechoic and also hypoechoic hepatic parenchyma changes [15]. This finding is at variance with the study from central Africa where intrinsic mass lesions, namely AIDS- related lymphoma, Kaposi sarcoma of the liver, diffuse nodular regenerative hyperplasia, multiple hyperechoic nodules from extra pulmonary pneumocystic carinii, and mycetic abscess were found. The absence of theses hepatic changes in our patients may suggest an improved quality of life consequence to the administration of highly-Active Antiretroviral Therapy HAART. There was however sonographic evidence of increased liver parenchyma echogenicity $(26.04 \%)$ of these patients. This latter is compatible with well documented fatty changes in AIDS patients.

Splenomegaly was demonstrated in $(31.25 \%)$ of the patients. Other abnormalities of the spleen in this study occurred far less common. The four cases of focal hypoechoic splenic areas may have been due to splenic lymphoma and small abscesses. Focal splenic lymphomas are commonly depicted as a hypoechoic lesion and were often seen in association with splenomegaly as in the cases identified. There was one case of echogenic foci may due to splenic calcification. There were $3(3.12 \%)$ hypoechoic splenic parenchyma, and $5(5.21 \%)$ cases of increased echogenicity of splenic parenchyma. These cases of splenic hyperechogenicity could not be attributed to any particular disease entity.
The frequency of splenomegaly was compared with that recorded by Tshibwabwa et al [12] in which $(35 \%)$ of their patients had splenomegaly and this agreed with the findings of this study. Also slightly matched the results of Objimi et al[2] in which identified splenomegaly in (45\%) of all samples.

Splenomegaly without focal lesion is relatively common in the tropics and could have myriads of causes including malaria, septicaemia, typhoid, schistosomiasis, portal hypertension, haemolytic anaemia and tropical splenomegaly [16].

Lymphadenopathy was diagnosed in 13 patients (13.54\%) in this study enlarged lymphnodes were seen as multiple and were greater than 1 $\mathrm{cm}$, mostly oval shaped with an echogenic hilum and a narrow symmetric cortex suggesting that they were benign. An ultrasound guided fine needle aspiration could have further characterized these nodes, but this could not be carried out in the centre because of unavailability of appropriate needles and other facilities. This record disagrees with Langer who studied [17] abdominal sonographic findings in patients with AIDS, and he found that lymphadenopathy was (21\%), N'Zi Pk et al recorded [18] that lymphadenopathy was $(17.2 \%)$, and this approximately agreed with this study.

The gallbladder wall was thickened in 4 (4.17\%) patients; cases of cholelithiasis were not seen. These patients had no symptoms referable to the biliary system and the cause of this finding remains unknown.

Enlargement of the kidney was seen in 1 case bilaterally and a small kidney also in 1 case. The kidney size is not significant in our study. This disagreed with the study carried by Blessing et al [19] which showed that renomegaly was seen in (18.7\%). The difference may due to the difference in the sample size which was less in our study.

There were $4(4.17 \%)$ of patients had decreased renal cortical echogenicity and $5(5.21 \%)$ of patients had increased renal cortical echogenicity. Other renal findings included 4 $(4.17 \%)$ case of AIDS nephropathy and 10 $(10.41 \%)$ case of renal stones mainly right side, this study showed significantly increased renal stone, but still the detection of nephrolithiasis is a coincidental finding because there was no correlation between HIV infection and formation of renal stones. This strongly agreed with the 
findings of Tshibwabwa et al [13] and Objimi et al [2].

The pattern of increased renal cortical echogenicity seen in this study was similar to that described by Herald et al [20] as AIDS nephropathy.

\section{Conclusion}

A wide range of abnormal abdominal organs can be seen on ultrasonography in patients with AIDS. The most frequent findings include splenomegaly, enlarged lymph nodes, hepatomegaly and increased liver echogencity. Focal lesions of parenchyma of the organs, nephromegaly, ascites, portal hypertension and abscesses are uncommon. The study's data supports the fact that the ultrasound is useful diagnostic tool for AIDS patient.

Funding: No funding resourses .

Conflicts of interest: The authors report no conflicts of interest. The authors alone are responsible for the content and writing of the paper.

Ethical approval: Approved.

\section{REFERENCES}

1. Vinay Kumar, Ramzi S. Cortan, Stanley L. Robbins. Basic Pathology. $7^{\text {th }}$ ed., Saunders; Philadelphia; Pennsylvania: 2003; 156-147

2. Millicent O Obajimi, Mojisola O Atalabi, Godwin Ogbole, Adenike T Adeniji-Sofoluwe, Atinuke M Agunloye, Ademola J Adekanmi ; et al Abdominal Ultrasonography in HIV/AIDS Patients in Southwestern Nigeria, BMC medical imaging: 2008;8,1-6

3. Roderick N M Mac Sween and Keith Whaley, Muir's Textbook of Pathology, $1^{\text {3th }}$ ed., Arnold; London: 1992;3 p.p 243

4. Epidemiological Fact Sheets on HIV and AIDS, www.who.int.globalatlas/predefined 2008. (Accessed 12 November 2011).

5. www.who.int. 2010 report.(Accessed 10 October 2011).

6. www.who.int/hiv/data. 2009. (Accessed 25 October 2011).

7. http://www.unaids.org/en/ (Accessed 12 December 2012).

8. Kathryn A. Gill. Abdominal ultrasound. W.B. Saunders; USA: 2002 chap 2, 35-56
9. Syed Amir Gilani. Guidelines and Protocols for Medical Diagnostic Ultrasound. $1^{\text {st }}$ ed., The Burwin Institute of Ultrasound. Lahore, Pakistan: 2002; 44-46

10. Miller F, Patrikh S, Gore R, Nemcek A, Fitzgerald S, Vogelzang R: Renal Manifestations of AIDS. RadioGraphics 1993; 13:587-596.

11. Devin Dean. Ultrasonography of the Abdomen and Small Parts. The Burwin Institute of Diagnostic Medical Ultrasound; Lunenburg, Canada: 2005; 44

12. Carol M. Rumack, Stephanie R. Wilson, J. William charponeau. Diagnostic Ultrasound. Vol. 1,2; Mosby; USA: 1991; 10-230

13. ET .Tshibwabwa, P. Mwaba, J Bogle-Taylor, A, http://www.ncbi.nlm.nih.gov/pubmed?term=Zum la\%20A\%5BAuthor\%5D\&cauthor=true\&cauthor _uid=10823454. Four Years Study of Abdominal Ultrasound in 900 Central Africa Adults with AIDS referred for Diagnostic Imaging. Pub Med Abdom Imaging: 2000; 25:290-296.

14. Townsend RR. CT of AIDS-related Lymphoma. Am J Roentgenol: 1991; 156:969-974.

15. Yee JM, Raghavendra BN, Horii SC, Ambrosino M. Abdominal Sonography in AIDS: a review. $J$ Ultrasound Med 1989; 8: 705-714.

16. Schneiderman DJ. Hepatobiliary Abnormalities of AIDS. Gastroenterrol Clin North Am: 1988; 615-630.

17. Richard M. Gore, Frank H. Miller, Vahid Yaghami. Seminars in Ultrasound, CT and MRI. Science direct. 1998. 19:175-189.

18. Blessing Ose-Emenim Igbinedion. Journal of radiology. Benin, Nigeria: 2009.

19. Herald T. Lutz \& Hassen A. Gharbi. Manual of Diagnostic Ultrasound in Infectious Tropical Diseases. Springer-verlag; Berlin Heidelberg, Germany: 2006; 10, 72-80.

20. Langer R, Langer M, Schütze B, Zwicker C, Wakat JP, Felix R . Abdominal Sonographic Findings in Patients with AIDS, Rontgenblatter,PubMed : 1989;22:121-125

21. N'Zi PK, Coulibaly A, N'Dri K, Quattara ND, Diabate SA, Zunon-kipre E, Djedje AT: Ultrasound aspects of abdominal involvement in adults with HIV infections in the Ivory Coast: apropos of 146 cases. Sante 1999; 9:85-88.

Peer reviewer: Hala Ismail ;Assistant Professor of Tropical Medicine,Zagazig University,Egypt.

Editor :Tarik Zaher;Assistant Professor of Tropical Medicine,Zagazig University,Egypt 